\title{
Análise Bioética frente a Covid-19: abordagem necessária para a área da saúde
}

\author{
Bioethical analysis against Covid-19: a necessary approach for the health area \\ Análisis bioético contra Covid-19: un enfoque necesario para el área de la salud
}

Recebido: 20/01/2022 | Revisado: 27/01/2022 | Aceito: 02/02/2022 | Publicado: 04/02/2022

Alessandra Conceição Leite Funchal Camacho
ORCID: https://orcid.org/0000-0001-6600-6630
Universidade Federal Fluminense, Brasil
E-mail: alessandracamacho@id.uff.br
Victor Hugo Gomes Ferraz
ORCID: https://orcid.org/0000-0003-4784-449X
Universidade Federal Fluminense, Brasil
E-mail: victorferraz@id.uff.br
Juliana de Oliveira Nunes da Silva
ORCID: https://orcid.org/0000-0002-9111-3806
Universidade Federal Fluminense, Brasil
E-mail: juliana_nunes@id.uff.br
Suellen de Almeida Barroso
ORCID: https://orcid.org/0000-0003-1947-8648
Universidade Federal Fluminense, Brasil
E-mail: suellenalmeida @id.uff.br
Vitória Meireles Felipe de Souza
ORCID: https://orcid.org/0000-0002-1129-6324
Universidade Federal Fluminense, Brasil
E-mail: vifelipe@id.uff.br

\section{Resumo}

Este artigo apresenta como objetivo analisar as questões bioéticas na área da saúde frente a Covid-19. É uma proposta de reflexão, com abordagem qualitativa do tipo descritiva sobre a análise bioética frente a Covid-19 num diálogo baseado nos princípios que a contextualizam que são: a beneficência, a autonomia e a justiça. Este artigo está pautado nas seguintes discussões: as questões bioéticas sobre a violência contra o idoso na Pandemia da Covid-19; a autonomia do cliente oncológico na ótica da bioética durante a Pandemia da Covid-19 e a análise bioética na Pandemia da Covid-19. Os desafios da bioética em relação as questões relacionadas ao direito a autonomia e o acesso aos serviços de saúde enunciam problemas de saúde pública no panorama brasileiro mas, destacam um alerta necessário para repensar as práticas de cuidado a população através das políticas públicas. Para ampliar as discussões recomenda-se pesquisas sobre a Covid-19 com um enfoque bioético sem a pretensão de esgotar mas, ampliar as discussões, evidenciando outras temáticas relevantes sobre a Covid-19 no que diz respeito a dignidade humana.

Palavras-chave: Enfermagem; Pandemias; Coronavirus; Bioética; Saúde.

\begin{abstract}
This article aims to analyze the bioethical issues in the health area facing Covid-19. It is a proposal for reflection, with a qualitative descriptive approach, on bioethical analysis against Covid-19 in a dialogue based on the principles that contextualize it, which are: beneficence, autonomy and justice. This article is based on the following discussions: bioethical issues about violence against the elderly in the Covid-19 Pandemic; the autonomy of the cancer client from the perspective of bioethics during the Covid-19 Pandemic and the bioethical analysis in the Covid-19 Pandemic. The challenges of bioethics in relation to issues related to the right to autonomy and access to health services enunciate public health problems in the Brazilian panorama, but highlight a necessary warning to rethink the population's care practices through public policies. To broaden the discussions, research on Covid-19 with a bioethical approach is recommended without intending to exhaust but broaden the discussions, highlighting other relevant themes about Covid-19 with regard to human dignity.
\end{abstract}

Keywords: Nursing; Pandemics; Coronavirus; Bioethics; Health.

\section{Resumen}

Este artículo tiene como objetivo analizar cuestiones bioéticas en el área de la salud frente al Covid-19. Se trata de una propuesta de reflexión, con un enfoque descriptivo cualitativo, sobre el análisis bioético frente al Covid-19 en un diálogo basado en los principios que lo contextualizan, que son: beneficencia, autonomía y justicia. Este artículo se basa en las siguientes discusiones: cuestiones bioéticas sobre la violencia contra las personas mayores en la pandemia Covid-19; la autonomía del paciente con cáncer desde la perspectiva de la bioética durante la pandemia Covid-19 y el 
análisis bioético en la pandemia Covid-19. Los desafíos de la bioética en relación a temas relacionados con el derecho a la autonomía y el acceso a los servicios de salud enuncian problemas de salud pública en el panorama brasileño, pero destacan una advertencia necesaria para repensar las prácticas de atención de la población a través de políticas públicas. Para ampliar las discusiones, se recomienda la investigación sobre Covid-19 con un enfoque bioético sin pretender agotar sino ampliar las discusiones, destacando otros temas relevantes sobre Covid-19 con respecto a la dignidad humana.

Palabras clave: Enfermería; Pandemias; Coronavirus; Bioética; Salud.

\section{Introdução}

Com a pandemia da COVID-19 declarada em 2020 ocorreu a elevada proporção de pessoas acometidas que necessitavam de internação hospitalar prolongada. As demandas dessa enfermidade aumentou o uso de recursos já escassos, tem exigido uma demanda extraordinária e sustentada por cuidados de saúde que não pode ser adequadamente satisfeita por sistemas de saúde saturados nos levando a repensar os aspectos éticos relacionados a Covid-19 (Recuero, 2020).

As questões relacionadas a autonomia do indivíduo se aprofundaram ainda mais durante a Pandemia da COVID-19 através das análises bioéticas, envolvendo todos os aspectos de nossas vidas e condicionando debates. A chegada do coronavírus revelou muitos problemas que já existiam: sistema de saúde precário, desigualdade social e distaciamento das ações do estado. É preciso reconhecer que vivenciamos um cenário incontrolável onde o vírus teve enorme repercussão no mundo.

Neste sentido, se inseriu um conflito sobre o benefício coletivo (por meio da adoção de medidas de distanciamento social) e o isolamento social abrindo espaço para a reflexão bioética e criando um ambiente propício para discutir e aperfeiçoar práticas de cuidados na área da saúde. O que sabemos é que não existe resposta correta para o problema da Pandemia da Covid-19 a não se condutas voltadas para o coletivo, sendo a abordagem mais viável encontrar informações tangíveis que sejam compreendidas e realizadas pela população (Nora, 2021).

Nas interlocuções bioéticas de seus princípios durante a Pandemia da Covid-19, a aplicação dos quatro princípios (beneficência, não maleficência, autonomia e justiça) depende das condutas éticas das pessoas envolvidas, bem como o bom senso no cotidiano que vislumbramos através da capacidade de discernimento de cada indivíduo. É uma dependência que "flexibiliza" esses preceitos, que não podem ser aplicados de maneira individual e sem a análise das especificidades das situações (Sanches, Cunha, Siqueira \& Siqueira, 2020).

Em detrimento da liberdade individual para controlar a pandemia de covid-19 através da imposição do distanciamento social as questões bioéticas não se esgotam e envolvem uma discussão voltada para o bem coletivo com o objetivo de evitar danos em demasia para a população. Ou seja, cada pessoa tem o seu livre arbítrio desde que essa decisão não prejudique outros (Nora, 2021).

Em outra análise há os profissionais da saúde que necessitam considerar a ciência para a tomada de decisão ética, com a intencionalidade de melhor assistir. Esse assistir realizado pelos profissionais para o coletivo à saúde é orientado nos casos suspeitos quanto a distanciamento social e reconhecimento dos sinais e sintomas característicos, bem como as normas de cuidado principalmente quanto ao uso de máscara. O mais importante é não fazer uma análise bioética diminuta que induzam a deliberação a partir de um único princípio, mas que leve em consideração todo o contexto (Nora, 2021).

A bioética como campo de análise transdisciplinar nos permite delinear a evolução tecnológica entendendo os dilemas que permeiam a sociedade, gerando uma reflexão sobre a possibilidade de nos trazer respostas no enfrentamento a Covid-19. No que tange a seu arcabouço epistemológico há uma demanda dialógica em duas questões importantes que são a autonomia da pessoa e a importância em oferecer um suporte social diante das vulnerabilidades que se apresentam na esfera econômica, de assistência à saúde e informativa (Dadalto, 2021). 
Neste contexto, devemos reconhecer a própria declaração de emergência sanitária como pandemia numa escala global perpassando do prisma individual para uma escala coletiva, como grupos de pacientes ou cidadãos ou, ainda, a sociedade como um todo, que deve prevalecer na tomada de decisões principalmente no que tange sobre a Bioética (Recuero, 2020).

Certamente estamos passando por uma situação preocupante no que tange sobre a vulnebilidade do ser humano em seus aspectos sociais e econômicos em que o mundo não será o mesmo. A pandemia vislumbrou novas interfaces de análise bioética onde percebemos o quão vulneráveis somos e como estamos interconectados onde, coisas positivas também surgiram como a solidariedade, a visibilidade do ser humano como único e reavaliação dos serviços públicos de assistência a saúde.

Desta forma, este artigo tem como objetivo analisar as questões bioéticas na área da saúde frente a Covid-19.

\section{Metodologia}

É um artigo de reflexão que possui abordagem qualitativa do tipo descritiva sobre a análise bioética frente a Covid-19 na área da saúde. Entendendo o princípio da bioética da autonomia no respeito ao acesso aos serviços de saúde com uma diretriz baseada no diálogo através das reflexões oriundas da Pandemia da Covid-19.

A abordagem de análise deste artigo é do tipo qualitativa a partir de conteúdos coletadas no contexto das ocorrências através de fenômenos sociais se caracterizando como um estudo de caso com o propósito de descrever determinadas aspectos de interesse (Pereira, Shitsuka, Parreira, \& Shitsuka, 2018).

Além disso, a abordagem desse artigo prioriza a discussão direcionada na Agenda de Prioridades de Pesquisa do Ministério da Saúde, no seu Eixo 9 sobre Programas e Políticas em Saúde no seu item 9.27 que trata sobre a avaliação da oferta de ações e serviços de saúde da Atenção Básica frente às necessidades da população (Brasil, 2018).

Este artigo está pautado nas seguintes discussões: as questões bioéticas sobre a violência contra o idoso na Pandemia da Covid-19; a autonomia do cliente oncológico na ótica da bioética durante a Pandemia da Covid-19 e a análise bioética na Pandemia da Covid-19.

A proposta de discussão sobre estes tópicos não esgotam os temas abordados pela Bioética, pelo contrário, evidencia outras temáticas relevantes sobre a Covid-19 no que diz respeito a dignidade humana. No entanto, este artigo de reflexão serve de subsídio para outras perspectivas de análise sob a ótica da Bioética.

\section{Resultados e Discussão}

\section{As questões bioéticas sobre a violência contra o idoso na Pandemia da Covid-19}

Com a Pandemia da Covid-19 em 2020 foi evidenciado a necessidade dos idosos manterem o distanciamento social e realizar as recomendações das autoridades da área da saúde. Esse público foi o mais afetado, principalmente na presença de comorbidades, em relação a violência contra o idoso (Souza \& Mendes, 2021).

Infelizmente a residência deveria ser o lugar mais seguro nestas situações, no entanto, a violência do idoso tem no âmbito familiar uma ocorrência de importante análise a luz da bioética. No cenário brasileiro em virtude com a crise econômica e, justamente durante a Pandemia ocorreu um elevado número de demissões e consequentemente dificuldades financeiras por parte das famílias. Junto dessa situação em consonância com as demandas de cuidados aos idosos a violência se tornou uma tônica de quebra do elo de confiança entre os membros da família.

De maneira mais esclarecedora, especificamente no Brasil, o Ministério da Mulher, da Família e dos Direitos Humanos registrou cerca de três mil a oito mil denúncias entre março e abril de 2020 onde nesses meses houve as maiores taxas de isolamento social (Ministério da Mulher, da Família e dos Direitos Humanos, 2020). 
As características dessas denúncias são relacionadas a violência física, sexual (por idosas), abandono, psicológica, dentro de instituições e pelo estado (estrutural) com desrespeito aos direitos de acesso as políticas públicas. É verificado uma ampla característica dos tipos de violência e que não devem ser ignoradas pelos profissionais de saúde.

Para melhor compreensão dessa situação foi realizado um estudo no Distrito Federal, onde foi possível identificar os tipos de ocorrências de violência contra o idoso: sobrecarga do cuidador (77,4\%); filhos com ideação equivocada sobre a evolução doença de seus pais (27\%); afetividade negativa dos filhos para com os pais idosos (24,3\%) (Matos, Braz, Albernaz, Sousa Pinheiros \& Ferreira, 2021).

A Bioética neste aspecto analisa como relevante em relação ao idoso a importância deste se autodeterminar nas questões relacionadas ao plano familiar, mas também no âmbito cotidiano, entendendo que a autonomia é um direito irrevogável e deve ser contextualizado de acordo com a realidade do idoso (Oliveira, Machado \& Dadalto, 2020).

De fato, a violência em suas interfaces familiares exige uma análise específica de acordo com essa realidade, porque em alguns aspectos estão associados a cultura, a naturalização, ao sentimento de vergonha e até por medo em que o idoso se sente impedido de efetuar a denunciar. É uma realidade marcada por convivência e nem sempre esta é benéfica.

A discussão bioética voltada para a violência ao idoso se apresenta em nosso país com características privadas (invisibilizadas), nas instituições, sociais e políticas na ausência de acolhimento exigindo um planejamento das esferas governamentais e até mesmo nas unidades de saúde e pelas instituições jurídicas na observância do biodireito (Moraes, Marques, Ribeiro \& Souza, 2021).

Uma característica relevante é a violência voltada para o gênero onde as idosas estão mais vulneráveis, sendo evidenciada em nossa sociedade que retrata a desigualdade de gênero em diversas instâncias como no acesso ao emprego, a diferença de salário, as taxas elevadas de feminicídio e abuso sexual. Ou seja, são elementos importantes que se refletem na velhice que devem ser discutidos e fomentar medidas jurídicas plausíveis com vistas a prevenção e acolhimento para evitar problemas futuros (Sousa, Araújo, Souto, Santos, Santos \& Almeida, 2021).

Um estudo desenvolvido em São Paulo (Campinas) destaca a maior incidência de violência quanto ao gênero feminino, onde 1.217 idosos sofreram violência, sendo que 69,5\% eram do sexo feminino, na faixa etária entre 60 a 69 anos $(35,8 \%)$. Além disso, como informação relevante essa ocorrência foi por negligência (33,1\%) e na residência (92.9\%) em sua maior parte. Infelizmente a autoria da violência esteve ligada a homens $(55,6 \%)$ e do tipo corporal $(24,4 \%)$ (Lopes \& D`Elboux, 2021).

A vulnerabilidade física e social do idoso nos leva a considerar a importância da preservação da autonomia com vistas ao direito a dignidade humana. Neste aspecto se faz presente o princípio da Bioética da justiça na garantia ao acesso as políticas públicas protetivas. É preciso entender que num sentido mais amplo as instituições educacionais e aquelas voltadas para as mídias sociais divulguem informações que auxiliem a sociedade na conscientização sobre o direito aos serviços de assistência jurídica, social e de saúde (Moraes, Marques, Ribeiro \& Souza, 2021).

Com o isolamento da Pandemia da Covid-19 a bioética nos traz essas reflexões para produção de informações relevantes para proteção e prevenção da violência contra o idoso com vistas a educação em saúde através de ações voltadas a esfera pública trazendo a importância de desenvolver laços de afetividade que se perpetuem em cuidados no âmbito familiar. Sem dúvida também deve-se compreender a necessidade de uma rede de suporte que venha a ajudar o idoso, seus familiares e cuidadores num diálogo contínuo diante das dificuldades de acesso aos serviços públicos disponíveis.

\section{A autonomia do cliente oncológico na ótica da bioética durante a Pandemia da Covid-19}

A Pandemia da Covid-19 levantou um grande desafio sobre a melhor maneira de assistir o paciente com comorbidades dentre as quais aqueles na área oncológica. Além disso, também houve novas maneiras de repensar sobre a 
atuação dos profissionais de saúde diante aos cuidados a essa clientela em detrimento do coronavírus. Nessa perspectiva há também o desafio em evoluir e entender a que nos levou a novas descobertas e informações, podendo proporcionar mudanças e adaptações na assistência à saúde direcionada ao paciente com câncer e aos profissionais envolvidos nos cuidados (Nora, 2021).

Além da oportunidade de novas abordagens a essa clientela também foi determinante perceber a necessidade de compreender os diversos tipos de cânceres e estadiamentos com a infecção da Covid-19 em relação a especificidade do tratamento a ser desenvolvido (Galindo et al, 2021).

Em relação ao direito dos pacientes sob a ótica da bioética é relevante entender sobre os seus princípios que nos conduzem a reflexões importantes na assistência ao cliente oncológico. O princípio ético da autonomia é o mais discutido porque remete as especificidades das situações em detrimento do livre arbítrio da pessoa e essas questões devem ser objeto de análise num contexto diferenciado como o atual (Lima \& Biasoli, 2018).

O contexto atual em detrimento ao princípio da autonomia demonstra a necessidade de compreensão dos profissionais de saúde sobre uma decisão compartilhada, o respeito a dignidade da pessoa, o direito a informação e a responsabilidade do paciente, da família e da equipe multidisciplinar. Sem dúvida são discussões pertinentes que nos direcionam a efetividade das políticas públicas disponível a população (Dadalto, 2021).

Os dilemas bioéticos estão presentes na indicação sobre a melhor conduta deve sempre perpassar pelo paciente na análise das informações trocadas, pela família que se faz presente e assim como o paciente necessita de ajuda qualificada. Nessas instâncias deve prevalecer o acesso a todos os recursos necessários sem qualquer prejuízo ao direito a beneficência, a autonomia e a justiça (Cunha, Dantas, Santos, Comassetto \& Santos, 2021)

Essa efetividade é ainda vista como limitante no contexto da vida e em muitas situações cotidianas no acesso aos serviços de saúde que são precários e não atendem as demandas da população como um todo. Infelizmente ainda estamos numa análise reducionista que não dirimiu as desigualdades já existentes antes da pandemia e que com a pandemia da Covid19 esse problema se ampliou na discussão sobre quais são as melhores escolhas para o cliente oncológico (Camacho, 2021).

Uma outra questão é a centralidade do paciente com o respeito a sua autonomia e não nos profissionais de saúde. O relacionamento interpessoal nesta situação deve envolver o compromisso com o diálogo com a família e o paciente voltado para a melhor escolha de acordo com a individualidade e especificidade que cada caso remete. A rede de suporte deve evidenciar a melhor maneira de conduzir as escolhas evitando conflitos bioéticos.

No acesso aos serviços de saúde é preciso vislumbrar no cuidado holístico, voltado para o conforto e humanizado. Essa assistência deve orientar a conduta assistencial dos profissionais de saúde em que a decisão sempre esteja pautada no respeito aos princípios bioéticos baseada na autonomia (no respeito a decisão), na beneficência (numa assistência humanizada) e na justiça (com o direito ao acesso aos serviços de saúde) (Cardoso, Pacheco, Caldas, Souza, Peres \& Nunes, 2019).

As vulnerabilidades em diversos aspectos do paciente oncológico devem ser compreendido no acesso ao serviço de saúde, na realidade dos profissionais da saúde ou até mesmo no próprio combate a pandemia da Covid-19 com vistas a autonomia e o seu valor empreendido nas informações prestadas e no próprio relacionamento interpessoal partilhado (Dadalto, 2021).

O que é visualizado atualmente é a maneira dinâmica como as ocorrências acontecem e por isso as mediações dos conflitos bioéticos devem ser atenuados com estratégias de capacitação continuada para a equipe de saúde, amplo investimento do poder público na melhoria dos serviços de saúde, sociais e econômicos para que seja possível avançar no que é previsto na legislação brasileira (Camacho, 2021). 


\section{Análise bioética na Pandemia da Covid-19: interfaces em seus princípios}

Com a pandemia da Covid-19 surgiram novos diálogos e reflexões bioéticas importantes levando em consideração as desigualdades sociais agravadas pela pandemia, a dificuldade na confiança e transparência nas políticas governamentais e, principalmente na formulação de políticas próprias para as áreas essenciais de preocupação e interesse do ser humano (Nuffield Council on Bioethics, 2020).

Ficaram explícitas questões como as prioridades das pessoas para tratamento e cuidados, as liberdades das pessoas inclusive em relação ao isolamento social, o acesso a vacinação e proteção, além das complexas compensações envolvidas na tentativa de limitar o número de mortes por Covid-19. Daí também surgiram as disparidades na saúde, especialmente para aquelas pessoas com vulnerabilidade social e de extrema pobreza (Nuffield Council on Bioethics, 2020).

$\mathrm{Na}$ realidade brasileira a pandemia veio a evidenciar ainda mais as desigualdades sociais que já eram problemáticas no período que a antecedeu. A bioética em suas vertentes de análise nos trouxe a preocupação com o direito da população ao acesso aos serviços de saúde e sociais, bem como da própria equipe de saúde que se encontrou na linha frente na assistência complexa e especializada as pessoas enfermas com Covid-19 (Dadalto, 2021).

No contexto do biodireito há uma preocupação voltada para o princípio ético da justiça vislumbrado no acesso a melhores oportunidades de acesso ao emprego, moradia, a saúde, sociais e as políticas públicas que com a pandemia se agravaram a piora da assistência à saúde, a violência a criança, mulher e ao idoso, o desemprego se elevou ignorando a relevância da equidade populacional (Cândido, Alcântara \& Garrafa, 2020).

Outro aspecto que também ficou evidente na discussão bioética foram a ampliação da gravidade relacionada ao acesso dos pacientes no processo de diagnóstico e regulação para serviços de maior complexidade e a sua lentificação (Galindo et al, 2021). Associado a isso as pessoas em situação de vulnerabilidade social se encontraram residindo nas ruas por falta de emprego e de resolutividade imediata, onde tal fato nos levará a preocupantes análises epidemiológicas em estudo futuros.

Associada a estas questões a disponibilidade de vacinas no contexto bioético é entendido com um direito universal de todas as pessoas. A Organização Mundial da Saúde (OMS) defende tal acesso como um direito humano conquistado, no entanto, os laboratórios que investiram fortes recursos em pesquisas mostraram evidentes lucros com um mercado ávido ao seu fornecimento (Garrafa, 2020).

Atrelado a essa situação mundial, no panorama brasileiro ficamos estacionados no enfrentamento a Pandemia da Covid-19 com dificuldades na divulgação de informações importantes como a proteção individual no uso de máscaras e medidas de distanciamento social, além do amplo desprezo sobre a importância da imunização vacinal bem como a sua disponibilidade para atenuar os inúmeros casos e mortes por essa enfermidade (Garrafa, 2020).

Com o avanço do coronavírus a população mundial se viu em diversos dilemas com a velocidade de propagação, as estruturas dos sistemas de saúde comprometidos com a elevada demanda por leitos, equipamentos e respiradores com a necessidade de uma especialização até então desconhecida. Com a centralização dos recursos para essas demandas ainda foi aventada as chamadas escolhas pela vida ("Escolha de Sofia") nas instituições hospitalares, gerando impactos na relação médico-paciente, no direito a autonomia frente a tomada de decisão. Foi relevante analisar os protocolos de orientação para as tomadas de decisão publicados no Brasil, bem como as consequências psicológicas ocasionadas aos profissionais da área de saúde, sob a ótica da bioética (Torres, Félix \& Oliveira, 2020).

Um outro aspecto de análise bioética é sobre a população indígena em virtude das profundas desigualdades de atenção face à COVID-19. É oportuno salientar a existência do Subsistema de Saúde Indígena do Sistema Único de Saúde voltado para a assistência primária em territórios indígenas que, no entanto, foi deflagrada como lenta, pouco efetiva e desarticulada apontando para problemas direcionados a insegurança alimentar, a violência simbólica, econômica e assistencial (Santos, Pontes \& Coimbra Jr., 2020). 
O que é perceptível é a ameaça à comunidade frente a pandemia da Covid-19 em que os governos têm a obrigação ética de proteger e atenuar seu avanço em relação a população sob a sua responsabilidade. É relevante entender o bem comum como prevalente na sociedade mundial sendo esse o grande desafio de promover o bem-estar coletivo se tornando a justificativa comunitária para implementar medidas de saúde pública como vacinas, orientações de higiene e a manutenção no uso de máscara. Sem dúvida, o bom senso e o propósito comum deveriam prevalecer, mas infelizmente, isso não aconteceu. Muitos cidadãos foram motivados por uma liberdade equivocada que às vezes fragmenta o bem-estar comum, invocando perversamente a liberdade jurídica como sustento retórico para sua oposição ao bem da coletividade (Fins, 2021).

Em suas interfaces a bioética possui diversos alcances de discussão que precisamos avançar em pesquisas que visam atenuar desigualdades, priorizar oportunidades e favorecer reflexões voltadas para o direito a dignidade humana. O que vemos são assuntos que dizem respeito a proteção no direito a autonomia com a disponibilidade de recursos e uma rede de apoio que vai além dos discursos "prontos" e imediatistas mas provam a necessidade da equidade em seu princípio da justiça e que de fato traga benefícios reais a população em todas as regências situacionais sejam no âmbito mundial ou brasileiro.

\section{Conclusão}

Este artigo apresenta como objetivo analisar as questões bioéticas na área da saúde frente a Covid-19. Na perspectiva bioética vemos que temos muito que avançar nas discussões que já existiam no período anterior e se agravaram em detrimento a pandemia da Covid-19.

No cenário mundial ficou evidenciado a preocupação com disseminação da doença e a importância da visibilidade de seus atenuantes como o distanciamento social, o uso de máscara, a importância da vacinação bem como um acesso digno aos serviços de saúde. Além disso, também se tornou importante a questão dos grupos vulneráveis, sejam estes por estarem nos grupos com algum tipo de comorbidades ou por estarem em situação de pobreza.

Situações estas que também foram tônicas no cenário brasileiro e com alguns agravantes políticos, sociais e de saúde pública que que impossibilita o atendimento de todas as necessidades de saúde apresentadas pela população. A questões bioéticas tratadas neste artigo sobre a violência do idoso, a autonomia do cliente oncológico e as interfaces da bioética na pandemia da Covid-19 não teve a pretensão de esgotar esta temática, mas proporcionar como contribuição reflexões e novas pesquisas que denotem o protagonismo do ser humano em todas as suas especificidades existenciais.

\section{Referências}

Brasil. Ministério da Saúde (2018). Agenda nacional de prioridades de pesquisa em saúde (ANPPS). Brasília: Ministério da Saúde. http://bvsms.saude.gov.br/bvs/publicacoes/agenda_prioridades_pesquisa_ms.pdf

Camacho, A. C. L. F. (2021). O cliente oncológico em tempos de pandemia da Covid-19: uma análise bioética. Global Academic Nursing Journal.

Cândido, A. M., Alcântara, R. \& Garrafa, V. (2020). Secularismo, pós-modernidade e justiça na assistência à saúde em Engelhardt. Rev bioét, $28: 471$-78.

Cardoso, R. B., Pacheco, S. T. A., Caldas, C. P., Souza, P. A., Peres, P. L. P. \& Nunes, M. D. R. (2019). Prática confortadora ao idoso hospitalizado à luz da bioética. Rev bioét, 27(4):595-9.

Cunha, A. M. S., Dantas, H. L. L., Santos, S. A., Comassetto, I. \& Santos, R. M. (2021). Bioética e morte assistida: liberdade para morrer? Research, Society and Development, 10(6): e0510615435.

Dadalto, L (2021). Bioética e Covid-19. (2. ed.). São Paulo: Ed. Foco.

Fins, J. J. (2021). Love Thy Neighbor as Thyself: Building Community During Covid. London: The Hasting Center. https://www.thehastingscenter.org/lovethy-neighbor-as-thyself-building-community-during-covid/

Galindo, R. J. S. C., Andrade, L. V., Sena, G. R., Nogueira, L. R. M., Lima, T. P. F., Lima, J. T. O., et al (2021). Mulheres com câncer e COVID-19: letalidade e aspectos clínicos. Rev Bras Saúde Matern. Infant, 21(Supl. 1):S167-S175.

Garrafa, V. (2020). 2020 - Ano especialmente difícil: acesso às vacinas - direito universal ou objeto de consumo? Rev Bras Bioética;16 (e1):1-3.

Lima, C. V. C. \& Biasoli, L. F. (2018). Interfaces, lacunas e desafios entre bioética e oncologia. Rev bioét, 26(3):451-62. 
Research, Society and Development, v. 11, n. 2, e50511225952, 2022

(CC BY 4.0) | ISSN 2525-3409 | DOI: http://dx.doi.org/10.33448/rsd-v11i2.25952

Lopes, E. D. S. \& D’Elboux,, M. J. (2021). Violência contra a pessoa idosa no município de Campinas, São Paulo, nos últimos 11 anos: uma análise temporal. Rev Bras Geriatr Gerontol, 24(6): e200320.

Matos, N. M., Braz, M. C., Albernaz, E. O., Sousa, B. B., Pinheiros, H. A. \& Ferreira, D. T. T. (2021). Mediação de conflito: soluções propostas em atendimento a casos de violência contra a pessoa idosa. Rev Bras Geriatr Gerontol, 24(6):e210068.

Brasil. Ministério da Mulher, da Família e dos Direitos Humanos (2020). Relatório anual do Disque 100. Brasília: Ministério da Mulher, da Família e dos Direitos Humanos. https://www.gov.br/mdh/pt-br/centrais-de-conteudo/disque-100/relatorio-2019_disque-100.pdf

Moraes, C. L., Marques, E. S., Ribeiro, A. P. \& Souza, E. R. (2020). Violência contra idosos durante a pandemia de Covid-19 no Brasil: contribuições para seu enfrentamento. Ciênc. saúde coletiva, 25(Supl.2): 4177-4184.

Nora, C. R. D. (2021). Conflitos bioéticos sobre distanciamento social em tempos de pandemia. Rev. bioét, 29(1): 10-20.

Nuffield Council on Bioethics (2020). Novo diálogo público identifica áreas prioritárias para a formulação de políticas COVID-19: abordar as desigualdades, construir a confiança pública e envolver o público. Londres: Nuffield Council on Bioethics. https://www.nuffieldbioethics.org/news/newpublic-dialogue-identifies-priority-areas-for-covid-19-policy-making-addressing-inequalities-building-public-trust-and-involving-the-public

Oliveira, A. S. V., Machado, J. C., \& Dadalto, L. (2020). Cuidados paliativos e autonomia de idosos expostos à covid-19. Rev bioét, 28(4): 595-603. Pereira, A. S, Shitsuka, D. M., Parreira, F. J., \& Shitsuka, R. (2018). Metodologia da pesquisa científica. UFSM, NTE.

Recuero, M. (2020). La compartición internacional de datos personales relativos a la salud en tiempos de la COVID-19: aspectos éticos y legales para el impulso de la necesaria cooperación. Rev Bio y Der, 50:133-148.

Sanches, M. A., Cunha, T. R., Siqueira, S. S., \& Siqueira, J. E. (2020). Perspectivas bioéticas sobre tomada de decisão em tempos de pandemia. Rev. bioét, 28 (3): 410-417.

Santos, R. V., Pontes, A. L. \& Coimbra Jr, C. E. A. (2020). Um "fato social total”: COVID-19 e povos indígenas no Brasil. Cad. Saúde Pública, 36(10):e00268220.

Sousa, R. C. R., Araújo, G. K. N., Souto, R. Q., Santos, R. C., Santos, R. C. \& Almeida, L. R. (2021). Factors associated with the risk of violence against older adult women: a cross-sectional study. Rev. Latino-Am. Enfermagem, 29: e3394.

Souza, E. R. \& Mendes, T. C. O. (2021). Violência contra a pessoa idosa no contexto de pandemia pelo novo coronavírus. Rev. Bras. Geriatr. Gerontol, 24(6): e210079.

Torres, A.., Félix, A. A. A. \& Oliveira, P. I. S. (2020). Escolhas de Sofia e a pandemia de COVID-19 no Brasil: reflexões bioéticas. Rev Bio y Der, 50: 333 352. 